\title{
The Immune System - Complexity Exemplified
}

\author{
W.E. Paul ${ }^{1 *}$ \\ ${ }^{1}$ Laboratory of Immunology \\ National Institute of Allergy and Infectious Diseases \\ National Institutes of Health \\ Bethesda, MD 2082-1892, USA
}

\begin{abstract}
Keywords and phrases: immune system, complexity, diversity, tuning, response to perturbations, specificity, cellular/molecular environment, anatomic constraints
\end{abstract}

Mathematics Subject Classification: 92-02, 92B05

"... a riddle, wrapped in a mystery, inside an enigma" 1

With the exception of the nervous system, the immune system represents the most daunting example of complexity in biology. Indeed, it is a virtual poster child for complexity and, in many regards, the best arena in which to apply the increasingly powerful tools of computational analysis and modeling to achieve a predictive and quantitative understanding of function in the face of various perturbations.

In the immune system, complexity exists on at least three levels: first, there is the great diversity of differentiated states of the cells, from the highly varied lymphocytes, the bearers of the specificity of adaptive immunity, to the myriad cells that participate in innate immunity. Secondly, there is the state of responsiveness of these individual cells to the molecular mediators with which they deal, a phenomenon that can be subsumed under the term "tuning". Thirdly, there is the almost limitless diversity of the distinctive specificities of lymphocytes coupled with the much more limited, but still considerable, diversity of recognition elements displayed by the innate immune system. And all of this needs to be considered within an anatomical context. These highly interactive cells exist in virtually every bodily tissue and interact with the cells of those tissues in ways that regulate their function.

Listing all of the diverse cells of the immune system is surely unnecessary but perhaps a consideration of the branch points leading to one set of the various types of $\mathrm{T}$ lymphocytes can provide a taste of the great variety of these cells. $\mathrm{T}$ cells constitute a developmentally unified cell population that diversifies through a series of branches to give rise an ever increasing number of distinct cell types. The earliest of these diversification steps is that based on the decision as to the type of receptor for antigen the cells will employ. The more well studied $\mathrm{T}$ cells that are the dominant population in most mammalian species utilize T cell receptors (TCRs) composed of $\alpha / \beta$ chains while the second major T cell population, playing

${ }^{*}$ Corresponding author. E-mail: WPAUL@niaid.nih.gov

${ }^{1}$ Winston Churchill, 1939, but referring to the then Soviet Union, not immunology. 
important roles at mucosal surfaces and often displaying a much more stereotypical set of specificities, utilizes TCRs composed of $\gamma / \delta$ chains. The " $\alpha / \beta$ " T cells can then pass through a developmental branch point where they divide into CD4 and CD8 T cells. These T cells differ fundamentally from each other based on the class of major histocompatibility complex (MHC) molecules that acts to bind peptide fragments of antigen to create the cognate ligand recognized by the TCRs of these cells, with CD4 T cells utilizing the class II MHC molecules for this purpose.

The CD4 T cells can be divided into three broad lineages, conventional T cells, NKT cells and regulatory $\mathrm{T}$ cells (Tregs). Each lineage can be arrayed along a developmental pathway from so-called "single positive" thymocytes through the recent thymic emigrants to the peripheral lymphoid tissues to naïve CD4 T cells. Concentrating on the naïve conventional CD4 T cells, their encounter with their cognate antigen under appropriate conditions leads them to adopt one of several alternative functional phenotypes including the Th1, Th2, Th17, induced Treg and T follicular helper fates. Each one of these cell types, such as the Th1 cells, can exist as effector cells and distinct types of memory cells, at a minimum central memory cells and effector memory cells. Moreover, there is growing evidence that cells that have adopted one of the distinctive fates may alter their phenotype under particular stimulatory conditions to develop into yet another unique cell type, for example to a cell with properties of both Th1 and Th2 cells.

I have only listed the alternative fates along one pathway; the full range of possibilities of cells of the $\mathrm{T}$ lymphocyte lineage is far greater, not to mention the range of cells comprised within each of the other major immune cells types including B cells, innate lymphoid cells, dendritic cells, monocyte/macrophages and the various types of granulocytes, among others. Of course, the enumeration of distinctive cell types may well be an example of immunologic chauvinism, but these multiple cell types can be described because our knowledge of the molecules that distinguish the various cells of the immune system is extremely detailed. In turn, this is due to the often first use in immunology of some of the most powerful tools available to characterize cellular components, often for the very good reason that cells of the immune system are highly accessible and thus easy to study. Another point that bears comment upon is the degree to which the cells that have been designated as members of a particular type actually represent a fixed phenotype, fully distinct from their forebears and descendants, or whether they represent more of a continuum with the majority of the cells centered about the nodal point of the cell type.

A second, less widely appreciated, but still very important level of complexity is the state of responsiveness of each cell type. The term responsiveness here is a short-hand for a variety of different properties of the cell. This includes responsiveness and anergy ${ }^{2}$, which in turn may truly reflect a more general phenomenon that we have termed "tuning". Tuning is a parameter in which the past and current history of biochemical signals the cell has received/ is receiving determines the level of responsiveness of that cell to a new perturbation. Such a perturbation is mainly, but not completely, limited to engagement of the antigen-binding receptor, the $\mathrm{T}$ cell receptor for the $\mathrm{T}$ cell and the $\mathrm{B}$ cell receptor for the $\mathrm{B}$ cell. In addition engagement of cytokine receptors by their cytokine ligands constitutes an important set of perturbations. Tuning, although mainly a property of individual cells determined by their particular history, may also be determined by the state of inflammatory responses in the organism as a whole so that humans or animals with chronic infectious diseases may often be more sensitive to activation than would comparable cells in non-infected individuals.

The third and perhaps best appreciated of the states of complexity of lymphocytes, and one that is unique to the immune system, is the distribution of distinctive clonally distributed receptors for antigen of the B and T cells. Indeed, the antigen-specific receptors displayed by the lymphocytes may be the most characteristic feature of the immune system. Our understanding of receptor complexity rests on a long history of hard won knowledge. At the dawn of immunology, when Louis Pasteur first recognized that inoculating individuals with an attenuated or inactivated microbial pathogen would lead to protection against challenge with a virulent form of that pathogen, it was clear that the organism used for immunization and challenge had to be the same; the system was specific. But the range of diversity of the

\footnotetext{
${ }^{2}$ Anergy refers to a state of a lymphocyte in which it is alive but unable to respond to its cognate ligand even when the ligand is delivered under appropriate stimulatory conditions.
} 
receptors was not clear. Paul Ehrlich, the polymath who first presented a rational theory of immunologic specificity, envisaged a relatively small antigenic universe, since he believed that antigens were basically toxins that mediated their function and called forth responses because they interacted with preformed nutrient receptors on the cell surface.

This elegant concept had to be discarded when Karl Landsteiner, among others, showed that the antigenic universe was vast. A vast antigenic universe and a high degree of specificity required that the universe of antigen-specific receptors was similarly vast. How this could be incorporated into a concept that recognized the biology of the immune system, with both memory and tolerance, was only clarified a little over 50 years ago, in 1957, when David Talmage and Macfarlane Burnet independently proposed what we know call the "clonal selection theory". Clonal selection held that receptors for antigen preexisted in the individual but that they were distributed one to a cell. ${ }^{3}$ Thus, the breakthrough idea of Talmage and Burnet was that the selectable element of the immune system was the cell, not the receptor, and that the vast universe of distinctive receptors/ antibodies implied an equally vast universe of distinctive cells.

Thus, diversity of specificity/ cells brings with it many challenges for a suitable mathematical description of the immune system. We understand much of the molecular basis through which the diverse receptors are generated. Fundamentally, the immune system has evolved what amounts to a random peptide generator to insert distinct sequences in the region of the TCR and the BCR where they contact their cognate antigen. The system is capable of generating millions of distinct sequences. There is a considerable culling of the cells expressing receptors generated in this manner so that for $\mathrm{T}$ cells, only those with a modest to intermediate affinity for a self-peptide/ self MHC are allowed to migrate to the periphery, accounting in part for the immune system's tolerance of self-molecules. Even with this culling, the frequency of naïve $\mathrm{T}$ or B cell specific for a common antigen ranges between 1/100,000 and 1/1,000,000.

If the system had no controls, it might well mimic the behavior of a population of single cell organisms of considerable genetic diversity. Individual members would grow (or be eliminated) depending on their relative fitness to the environment, in this case fitness could be the affinity of their receptors for the antigen perturbing the system. Yet, despite the seeming similarity to an "unsupervised" selection system, there is growing evidence that the cells of the immune system are under rather precise control. Among the control mechanisms are both the capacity of cells of a given specificity to sense their relative numbers and to control their expansion based on these numbers and the existence of a specialized population of cells, Tregs, that will control the expansion/differentiation of conventional cells and do so in a manner that is at least partly based on immunologic specificity.

This description of key aspects of the immune system is hardly an exhaustive catalog of its complexity but one cannot omit a mention of the overarching issue of how the system adapts to its anatomic constraints. The immune system may often be studied in a tissue culture dish but it truly functions in a highly organized cellular structure in which it is continually receiving signals from its partners and from a wide array of so-called stromal cells that, far from being quiescent support cells, are deeply involved in an interactive set of molecular cues exchanged with the more classical cells of the system. Thus, the behavior of immune cells is highly colored by the cellular/molecular environment in which they exist and, indeed, by the means through which they migrate to these sites. Immune cells exchange signals of various classes with cells of the vascular tree as well as with the parenchymal cells of the tissue/organ.

Thus, the immune system offers challenges sufficient to test the growing power of mathematical attack on a biological problem. It is to the quantitative prediction of the outcome of given perturbations in the immune system that we envisage our mathematical/ modeling colleagues will apply themselves. There is a richness of opportunities and a myriad of challenges. Good luck!!!!!

\footnotetext{
${ }^{3}$ Actually, $\sim 1 / 3$ of $\mathrm{T}$ cells have two kinds of receptors
} 